wounding, extremes of temperature and even to pollination have all been demonstrated. Plants can be anaesthetized by chloroform, ether or even 1 per cent morphia. And in 1952, Loewy demonstrated the presence of actomyosin in slime moulds and proposed a theory of how actin and myosin could slide over one another to propel these organisms. This was before Hodgkin and Huxley put forward their Nobel-prizewinning model of animal muscle contraction.

But it is something of an affront to claim, as Simons does, that "plant movements are simply the manifestation of a deep-seated 'animalness' in plants". The many similarities between plants and animals are remarkable only because of our ignorance and zoocentric viewpoint. It would be more remarkable, in fact, if there was nothing similar in the cell chemistry and communication of multicellular plants and animals.

One of Simons' most interesting examples is the role of aspirin as a plant hormone. Aspirin, once extracted from willow tree (Salix) bark, is widespread in plants. Simons speculates that it may work in plants as it does in animals, knocking out pain-triggering prostaglandins and the closely related hormone jasmonic acid. Another mechanism is indicated by the relationship of salicylic acid to gentisic acid, the active ingredient of Ricca's factor, which is the trigger of electrical signals in the plant wound response.

Plants are exciting, however, not because of their similarities to animals but because of their differences. We are too easily seduced by the flashiness of rapid movement, but if we were able to see plants in a time-lapse film, we would see the most remarkable aspect of their behaviour: response to the environment not by movement but by growth. It is the remarkable plasticity of plant development that is thrilling. And the evolutionary consequences of this plasticity and the propensity of plants to clonal reproduction are intriguing. Some plants have lifetimes so long that they are almost immortal. Plants even challenge our concept of the cell, because plant 'cells' are connected by cytoplasmic strands forming a continuous network, a symplasm bounded by a single membrane reaching through the cell wall in fine pores, the plasmodesmata. The plant cell wall, for too long regarded as a kind of dead exoskeleton, is as much a part of the living cell as the plasmalemma.

Nevertheless, this is a very entertaining book, an easy and illuminating read. One area is not mentioned. What are the implications of the 'animalness' of plants for our eating habits? If a wave of electrical activity, reminiscent of pain and distress in animals, passes through a lettuce leaf as we bite into it, are salads to be banned, and can anyone recommend a humane way of killing a plant? $\square$

Martin Ingrouille is in the Department of Biology, Birkbeck College, Malet Street, London WC1E 7HX, UK.

\section{Ain't misbelieving}

Colin Howson

Bayes or Bust? A Critical Examination of Bayeslan Confirmation Theory. By John Earman. MIT Press: 1992. Pp. 265. $\$ 35, £ 31.50$.

SCIENTIFIC theories, even those dignified as laws, are logically underdetermined by the observational data on which they rest. A well-known philosopher's game is to produce, on request, a recipe for constructing an infinity of 'explanations' for any finite body of data inconsistent with the accepted scientific explanation. These are presumably not serious candidates for belief. But why not? Why do we regard some extrapolations of experience as more credible than others?

By the beginning of the nineteenth century, encouraged by the pioneering work of the English clergyman Thomas Bayes and by Laplace's even more remarkable extension of it, some people thought that they had the key to the answer: observational data endow hypotheses with probabilities which could (they thought) be straightforwardly computed by means of a theorem of the new mathematical theory of probability, a theorem now known, with some historical inaccuracy, as Bayes' theorem. All that remained was to do the calculations.

Unfortunately, the method by which the so-called prior probabilities in Bayes's theorem were determined, known in anglophone countries as the principle of indifference, depended on making fairly arbitrary decisions about the choice of appropriate possibility spaces. Different choices give different answers. Unsurprisingly, probabilism fell under increasing suspicion, until in the 1920 s and ' 30 s its condition was pronounced hopeless by, among others, the philosopher Karl Popper and the statistician R. A. Fisher.

But even as Fisher and Popper delivered their verdict, probabilism was finding a new and more modest role, as a theory of individuals' personal probability assignments subject to the sole constraint of consistency. The troublesome principle of indifference, or indeed any other for determining prior probabi- lities, is arguably more than a consistency constraint and is consequently disqualified. Slimmed-down, new-model probabilism, now called 'personalist Bayesianism', or just 'Bayesianism', nevertheless still provides, Bayesians claim, as much of a positive answer to the original question as is in principle possible. The evidence they cite in support of their claim is usually some variant of the following result. Where $\mathrm{H}$ is a set of hypotheses about data generated from a given source, all those probability distributions that initially assign the same members of $\mathrm{H}$ positive probability converge, as the data accumulate, in such a way that, for almost all data sequences, they assign the true hypothesis probability one in the limit.

Do convergence theorems such as this really offer even a partial solution of the original problem? Earman's excellent new monograph on Bayesian confirmation theory contains one of the few published discussions of this difficult question to be both lucid and authoritative. Possibly its most interesting and novel feature is that it reveals how those theorems relate to recent results in what is called formal learning theory. The same high standard of discussion is maintained in the rest of Earman's book which offers, among other things, a clear and accessible survey of the attempts to develop the basic principles of Bayesianism from conditions on acceptable bets; the extent to which the theory withstands the critical assaults that have been made on it; and how, taking as an example rivals to general relativity, in which field Earman is an expert, it copes with the grand scientific theories, which are much more than merely infinite extrapolations of finite data.

Earman's investigations lead to the deflation of some of the Bayesians' claims, and the qualification of others, although overall he still rates theirs the best among extant confirmation theories. If there is any complaint about this book, it is that it has a rather narrow focus, and such topics of current interest as calibration, invariant prior probabilities, upper and lower probabilities, the claims of the Dempster-Shafer theory, and Bayesian networks are not discussed. But it is the right of an author to select his material, and the manner in which Earman addresses those issues that do concern him merits only praise. His discussion is clear, even-handed and authoritative, and, not least important in a subject increasingly prone to technicality, conducted almost everywhere in readable and lively English.

Colin Howson is in the Department of Philosophy, London School of Economics, Houghton Street, London WC2A 2AE, UK. 